\title{
Oxytocin und die suchterhaltenden Mechanismen der Alkoholabhängigkeit
}

\author{
Till Faehrmann · Gerald Zernig · Sergei Mechtcheriakov (ID
}

Eingegangen: 8. Juni 2016 / Angenommen: 29. Mai 2017 / Online publiziert: 21. Juni 2017

(c) Der/die Autor(en) 2017. Dieser Artikel ist eine Open-Access-Publikation.

Zusammenfassung Eine der größten Herausforderungen in der Behandlung der Alkoholabhängigkeit ist die Aufrechterhaltung der Abstinenzfähigkeit der Patienten nach dem Abschluss der Entzugsbehandlung. Die erhöhte Stressempfindlichkeit und vielfältige Angstsymptome gelten als Hauptfaktoren, die das Alkoholverlangen triggern und Alkoholrückfälle begünstigen können. In den ersten Monaten der Alkoholabstinenz sind bei alkoholabhängigen Patienten häufig Schlafstörungen, erhöhte Reizbarkeit und depressive Verstimmungen als Indikatoren für die Aktivierung von Stressmechanismen zu beobachten. Hinzu kommt die Unsicherheit in den sozialen Interaktionen, die bei Abstinenzversuchen auftritt und oft zu stressassoziierten Erlebnissen führt. Dies resultiert häufig in der Vernachlässigung von sozialen Kontakten und stattdessen zunehmender Bevorzugung des Substanzkonsums sowie in der verminderten Fähigkeit, die für den Therapieerfolg notwendige soziale Interaktion mit dem therapeutischen Umfeld aufrechtzuerhalten.

Rezente Studien zeigen, dass das Peptidhormon Oxytocin sich durch eine anxiolytische Wirkung und die Fähigkeit, Stresssymptome abzumildern, auszeichnet.

\footnotetext{
T. Faehrmann · S. Mechtcheriakov ( $\square)$

Department Psychiatrie, Psychotherapie und

Psychosomatik, Medizinische Universität Innsbruck, Anichstraße 35, 6020 Innsbruck, Österreich

s.mechtcheriakov@i-med.ac.at

T. Faehrmann

Till.Faehrmann@student.i-med.ac.at

\section{G. Zernig}

Department Psychiatrie, Psychotherapie und Psychosomatik, Abteilung für Experimentelle Psychiatrie, Medizinische Universität Innsbruck, Anichstraße 35, 6020 Innsbruck, Österreich Gerald.Zernig@i-med.ac.at
}

Darüber hinaus entfaltet Oxytocin sog. prosoziale Effekte, welche zu einer verstärkten Hinwendung zu sozialer Interaktion führen. Intranasal lässt es sich zudem leicht und sicher verabreichen.

Aus neurobiologischer Sicht entfaltet Oxytocin seine anxiolytische Wirkung durch die Hemmung der Effekte des Corticotropin-releasing Factor (CRF) an GABAergen Interneuronen in Amygdala und Nucleus paraventricularis im Hypothalamus.

Somit kann die anxiolytische, stresshemmende und prosoziale Wirkung von Oxytocin für die Vorbeugung der durch Angstsymptomatik, Stressempfindlichkeit und sozialen Rückzug ausgelösten Rückfälle genutzt werden. Die neurobiologischen Grundlagen der Wirkungen von Oxytocin auf Mechanismen der Suchterhaltung bei Alkoholabhängigkeit werden in dieser Übersichtsarbeit im Detail vorgestellt und diskutiert.

Schlüsselwörter Alkoholabhängigkeit · Oxytocin · Angst · Stress · Amygdala

\section{Oxytocin and the mechanisms of alcohol dependence}

Summary One of the crucial purposes of treating alcohol-dependent patients is to enhance their ability to stay abstinent after detoxification therapy. Anxiety and stress vulnerability are the main factors provoking alcohol craving and relapse. In the first months of abstinence, alcohol-dependent patients frequently show sleep disturbances, irritability and depression, indicating chronic activation of stress pathways. In addition, the loss of confidence in interpersonal interactions results in social withdrawal and reduced willingness to participate in therapeutic programs.

Current research shows that the peptide hormone oxytocin exerts substantial anxiolytic effects and facilitates prosocial behavior. Oxytocin can be safely ap- 
plied as intranasal preparation. Oxytocin acts by inhibiting the effects of the corticotropin-releasing factor on GABAergic interneurons in the amygdala and paraventricular nucleus of hypothalamus.

Recent research strongly suggests that application of oxytocin may beneficially influence the mechanisms of relapse and craving by reduction of anxiety, stress vulnerability and social withdrawal in abstinent alcohol-dependent patients.

This article reviews neurobiological mechanisms of oxytocin effects on stress-related pathways and discusses the potential use of oxytocin in the treatment of alcohol addiction.

Keywords Alcohol dependence - Oxytocin · Anxiety · Stress · Amygdala

\section{Stress, Angst und reduzierte Abstinenzfähigkeit}

Eine der größten Herausforderungen in der Behandlung von Patienten mit Alkoholabhängigkeit ist die Erhaltung der Abstinenzfähigkeit. Die frühe Alkoholabstinenzphase, welche dem akuten Entzug folgt, ist durch eine erhöhte Vulnerabilität für Stress charakterisiert. Angst und Stress wurden als Schlüsselfaktoren für den Rückfall identifiziert [1]. Abstinente Patienten mit Alkoholabhängigkeit zeigen darüber hinaus eine Reihe an Symptomen wie Schlafstörungen, Angst, Reizbarkeit, Depression und Konzentrationsstörungen. Dieser Zustand persistiert noch lange über den akuten Entzug hinaus [1, 2].

Dem Rückfall geht zumeist das Phänomen des Cravings voraus [3]. Die Weltgesundheitsorganisation (WHO) definierte 1954 Craving als ein „dringendes und überwältigendes Verlangen oder unwiderstehlichen Impuls“, eine Substanz zu konsumieren [4]. Nach der Internationalen statistischen Klassifikation der Krankheiten und verwandter Gesundheitsprobleme (ICD-10) [5] ist Craving als diagnostisches Kriterium für Abhängigkeit gelistet und wird als starkes Verlangen oder Zwang, Drogen zu konsumieren, definiert. Von allen ICD-10-Kriterien für Alkoholabhängigkeit geht Craving mit dem höchsten relativen Risiko für eine Abhängigkeit nach einem Jahr einher [4]. Die Intensität des Cravings korreliert mit der Rückfallwahrscheinlichkeit [3, 4].

Die Beteiligung des Stresssystems bei Alkoholabhängigkeit ist vielseitig. Zum einen aktiviert die Gabe von Alkohol die Hypothalamus-HypophysenNebennierenrinden-Achse (,hypothalamic-pituitaryadrenocortical axis“, HPA-Achse), die der vorrangige Mediator der Stressantwort ist [2, 6, 7]. Zweitens erzeugt der Alkoholentzug physiologische Phänomene und Verhaltensänderungen, die mit Stressreaktionen verbunden sind und eine Aktivierung von Corticotropin-Releasing-Factor(CRF)-Neuronen im Gehirn nach sich ziehen [6, 8, 9]. Und drittens ist eine Stressexposition mit einem erhöhten Risiko der Wiederaufnahme des Alkoholkonsums (und damit Rückfälligkeit) bei abstinenten alkoholabhängigen Personen assoziiert $[6,10,11]$.

Obwohl die Wirkung von Alkohol individuell und variabel ist, wird es vorwiegend wegen seiner anxiolytischen Wirkung eingesetzt. Gleichzeitig ist der chronische Alkoholkonsum mit einer Anfälligkeit für Stress und Angst assoziiert [10], wofür die durch regelmäßige Aufnahme des Alkohols ausgelöste Neuroadaptation der Stresssysteme verantwortlich gemacht wird [10]. Koob und Le Moal verwendeten das Konzept der Allostase [12], um die Eskalation des Drogenkonsums bei Abhängigkeitserkrankungen zu erklären [13]. Allostase lässt sich als Adaptionsprozess definieren, der durch physiologische Anpassung an einen chronischen Stimulus - in diesem Falle Alkohol - eine funktionale Stabilität außerhalb der normalen Homöostase darstellt $[7,10,12]$. Dieser anfänglich adaptive Prozess führt mit der Zeit dazu, dass der Alkoholkonsum (z. B. im Rahmen eines Rückfalls) Stress erzeugt, der wiederum zu einem erhöhten Bedarf an weiterem Alkoholkonsum führt [11]. Dieser Teufelskreis, der eine positive Feedbackschleife darstellt, führt zu einer zunehmenden Allostase des Systems. Daraus resultiert eine Steigerung des Abhängigkeitsgrades sowie zunehmende Stressvulnerabilität [11].

Demzufolge wird Alkohol in Entzugsphasen getrunken, um die negativen Folgen des Entzugs zu verhindern, was zu einer Fortführung des Alkoholkonsums führt und gleichzeitig die Stresssymptomatik in der darauffolgenden Abstinenzphase weiter verschlimmert [10]. In der Tat zeigten seit 4 Wochen abstinente Alkoholiker bei Stressexposition mehr Angst und negative Emotionen als Reaktion auf Stress als die Kontrollgruppe, was infolgedessen zu verstärktem Craving führte [14].

\section{Amygdala und CRF bei Sucht}

Die endokrine Stressreaktion, die in Cortisolausschüttung mündet, nimmt ihren Ursprung im Nucleus paraventricularis („paraventricular nucleus“, PVN) im Hypothalamus. Dort wird in neuroendokrinen Zellen der CRF gebildet und von den neurosekretorischen Endigungen dieser spezialisierten Neuronen in die hypothalamisch-hypophysealen Portalgefäße ausgeschüttet. Über dieses Blutkapillarsystem gelangt CRF in den Hypophysenvorderlappen (,,anterior pituitary gland"), wo es zur Abgabe des adrenocorticotropen Hormons (ACTH) in den Blutkreislauf führt. ACTH stimuliert in der Nebennierenrinde die Synthese von Glucocorticoiden wie Cortisol [15]. CRF-Neurone finden sich auch in der Amygdala und werden ebenfalls durch Stress aktiviert [16]. Die CRF-Neurone in der Amygdala besitzen Glucocorticoidrezeptoren, weshalb sie möglicherweise zusätzlich durch die Nebennierenrinde mittels einer Rückkoppelungsschleife moduliert werden [16]. Furcht (Reaktion auf bestimmte Reize) und Angst (im Sinne eines $\mathrm{Zu}$ standes; ungerichtet) werden maßgeblich über die 
Amygdala vermittelt und reguliert [16-21]. Furchtund angstrelevante neuronale Efferenzen entspringen den zentralen [22], lateralen und basolateralen (basalen) Kerngebieten der Amygdala [17, 19]. Dabei ist zu beachten, dass die Nomenklatur der Amygdalasubregionen nicht einheitlich ist [23, 24].

Durch elektrische Stimulierung der zentralen Amygdala konnten bei Menschen artifiziell starke realistische Gefühle von Angst oder Furcht sowie Aktivierung des autonomen Nervensystems ausgelöst werden [16]. Vergleichbare Reaktionen wurden auch bei wachen Tieren durch chemische und elektrische Stimulierung der zentralen Amygdala hervorgerufen [16].

CRF-Neurone aus der zentralen Amygdala enden in einer Vielzahl von Hirnregionen, die zum Teil sehr unterschiedliche Funktionen erfüllen, wie z.B. im Nucleus striae terminalis („bed nucleus of the stria terminalis“, BNST, ein Teil der sog. „extended amygdala") [25, 26], im lateralen Hypothalamus, in der mesenzephalen retikulären Formation, im mesenzephalen Grau, in den lateralen und medialen parabrachialen Nuclei, im mesenzephalen Nucleus des Nervus trigeminus und im dorsalen Vaguskomplex [9, 12]. Bemerkenswerterweise innervieren Neuronen aus der zentralen Amygdala und dem Hirnstamm CRFNeurone im PVN und im lateralen Hypothalamus [16] und bilden damit eine Rückkopplungsschleife. Es lässt sich somit feststellen, dass der CRF sowohl im PVN als auch in der Amygdala einen anxiogenen Effekt ausübt [15].

CRF ist also anxiogen. Deshalb können CRF-Antagonisten die Verhaltensänderungen und physiologischen Auswirkungen von Stress hemmen [2]. Die Amygdala ist ein wichtiges Hirnareal, in dem die $\mathrm{Zu}$ nahme der CRF-Aktivität durch die anxiogenen Effekte des Alkohols zu lokalisieren sind. Im akuten Alkoholentzug ist eine gesteigerte Freisetzung von CRF in der zentralen Amygdala zu verzeichnen [7, 27]. Diese veränderte Reaktion auf Stress in der zentralen Amygdala bleibt bei Alkoholabstinenz auch nach dem akuten Entzug erhalten [27]. In Tierstudien wurde gezeigt, dass die lokale Gabe eines CRF-Rezeptorantagonisten in die zentrale Amygdala den anxiogenen Effekt des Alkoholentzugs bei Ratten verhindern kann [2, 7].

Auch in der gesteigerten Sensibilität für Stress spielt CRF eine wichtige Rolle. In weiteren Tierexperimenten zeigten alkoholabhängige Ratten, welche seit 6 Wochen alkoholabstinent waren, im Elevated-PlusMaze(EPM)-Test nach Stressexposition signifikant stärkeres Angstverhalten im Vergleich zur Kontrollgruppe. Durch eine vorausgehende Verabreichung eines CRF-Rezeptorantagonisten in die zentrale Amygdala konnte das gesteigerte Angstverhalten verhindert werden [7, 9]. Dadurch konnte gezeigt werden, dass CRF eine Regulationsfunktion der in der Alkoholabstinenz persistierenden gesteigerten Stressvulnerabilität zukommt [8].
Wurde alkoholabhängigen Ratten nach 3-5 Wochen Abstinenz wieder freier Zugang zu Alkohol gewährt, konnte die getrunkene Alkoholmenge durch die direkte Gabe eines CRF-Antagonisten in die zentrale Amygdala verringert werden [9].

\section{Oxytocin und Angst}

Oxytocin ist ein Peptidhormon, welches sich durch eine Vielzahl an Auswirkungen auszeichnet. Darunter fallen die anxiolytische Wirkung und die Abmilderung von Stress durch Oxytocin, welche in zahlreichen Studien untersucht wurden. Eine umfangreiche Übersicht dazu liefern Lee et al. [28].

Das Nonapeptid Oxytocin ist nach den altgrie-


„schnelle Geburt" bedeutet, da es mit der uteruskontrahierenden Wirkung von Oxytocin zunächst in Verbindung gebracht wurde [29]. Oxytocin wird vornehmlich in den hypothalamischen Kernen PVN und Nucleus supraopticus (SON) produziert [29]. Die meisten Axone enden in der Neurohypophyse, wo Aktionspotenziale die Oxytocinfreisetzung in den Blutkreislauf triggern, um die peripheren Wirkungen auszulösen. Man unterscheidet im PVN zwischen zwei unterschiedlichen Neuronensubpopulationen: den magnozellulären und den parvozellulären Neuronen. Die magnozellulären enden in der Neurohypophyse zur Freisetzung in die Blutbahn, die parvozellulären Neuronen hingegen terminieren in anderen Hirnarealen, darunter der Amygdala [29].

Oxytocin lässt sich als Anxiolytikum wirksam einsetzen. Dies ist in zahlreichen Studien gut belegt [28]. Die erste Tierstudie, welche bei Ratten einen anxiolytischen Effekt von Oxytocin nachweisen konnte, wurde 1997 von Windle et al. durchgeführt [30]. Die Autoren konnten zeigen, dass Oxytocin in der Lage ist, sowohl die Cortisolausschüttung als auch Angstverhalten zu verhindern. Oxytocin hat zweierlei Angriffspunkte in Bezug auf die Stressreaktion bei Angst: auf der einen Seite die endokrine Stressreaktion, auf der anderen Seite das Angstverhalten [31].

\section{Oxytocin und PVN}

Oxytocin wird zentral im PVN als Reaktion auf Stress ausgeschüttet, um die Stressreaktion abzudämpfen [32]. Auf die peripheren Oxytocinspiegel hat Stress vermutlich keine Auswirkung. Im Tierversuch konnte im Einklang damit gezeigt werden, dass Oxytocin auf der Ebene der Nebennierenrinde keinen Einfluss auf die Cortisolausschüttung hat. Es stellte sich heraus, dass der hemmende Effekt von Oxytocin im PVN stattfindet. Durch intracerebroventrikuläre (i.c.v.) Gabe von Oxytocin konnte die stressinduzierte Aktivitätssteigerung der HPA-Achse gehemmt werden [31, 32]. Der Zusammenhang zwischen peripherem Oxytocinspiegel und der intrazerebralen Freisetzung von Oxytocin ist jedoch noch Gegenstand der For- 




Abb. 1 Oxytocin hemmt die CRF-Neuronen des PVN über GABA-Interneurone, wodurch in der Folge die HPA-Achse inhibiert wird. ACTH adrenocorticotropes Hormon, CRF Corticotropin-releasing Factor, GABA $\gamma$-Aminobuttersäure, OXT Oxytocin, $P V N$ Nucleus paraventricularis

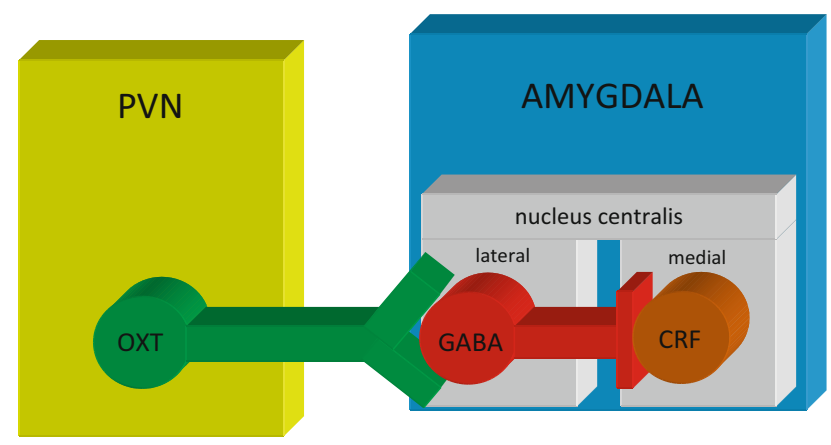

Abb. 2 Oxytocin hemmt CRF-Neurone im medialen Anteil der zentralen Amygdala über GABAerge Interneurone der lateralen zentralen Amygdala. CRF Corticotropin-releasing Factor, GABA $\gamma$-Aminobuttersäure, OXTOxytocin, $P V N$ Nucleus paraventricularis

schung und wird kontrovers diskutiert [33]. Darüber hinaus weisen die Oxytocin-mRNA- und CRF-mRNAExpression im PVN nach akuter und auch chronischer Stressexposition eine negative Korrelation auf. Hohe Oxytocinspiegel sind demnach mit erniedrigten CRFSpiegeln assoziiert [32].

Die große Mehrheit der neuronalen Antworten auf Oxytocin sind exzitatorischer Natur. Daher liegt der Schluss nahe, dass die Hemmung der CRF-Neuronen nicht auf direktem Wege erfolgt [34]. Intracerebroventrikuläre Gabe von Oxytocin hemmt die CRF-Expression im PVN [30, 32, 34]. Dieser Effekt kann durch die Gabe eines $\gamma$-Aminobuttersäure(GABA)-Antagonisten verhindert werden [34]. Morphologische Analysen offenbarten, dass GABA-Neuronen im PVN in Kontakt mit CRF-Neuronen stehen. Diese GABA-Neuronen werden bei Stress aktiviert und sind in die Hem- mung der Stressantwort eingebunden. Daraus folgt, dass Oxytocin über GABAerge Interneurone die CRFNeuronen des PVN hemmt [34]. Dieser postulierte Zusammenhang konnte in weiteren Untersuchungen bestätigt werden [35]. Die Folge ist eine verringerte Aktivierung der HPA-Achse (s. Abb. 1).

\section{Oxytocin und Amygdala}

Die Amygdala konnte in fMRI-Studien als einer der Kernpunkte der Oxytocinwirkung eingegrenzt werden [17]. Die anxiolytische Wirkung von Oxytocin in der Amygdala läuft wie im PVN ebenfalls über Interneurone ab [36]. Oxytocin hat exzitatorische Wirkung auf GABA-Interneurone im lateralen Teil der zentralen Amygdala [22]. Diese GABA-Neuronen inhibieren efferente Neurone im medialen Teil der zentralen Amygdala [22, 36, 37]. Die durch Oxytocin aktivierten GABAergen Interneuronen hemmen Neurone, die von dem medialen Teil der zentralen Amygdala (CeM) ausgehen und wahrscheinlich Furcht und/oder Angst modulieren (s. Abb. 2), [16, 36, 38]).

\section{Oxytocin im Einsatz gegen Suchtmechanismen}

Das Neuropeptid Oxytocin wirkt anxiolytisch [28, 31]. Der Angriffspunkt in der Amygdala ist dabei die Hemmung der angstassoziierten Efferenzen aus dem zentralen Kerngebiet [15, 22]. CRF stellt, wie in Abschn. „Amygdala und CRF bei Sucht“ dargelegt, ein anxiogenes Peptid dar, das in der zentralen Amygdala durch Angstreaktionen seine Wirkung entfaltet [2, 8, 9, 16, 27, 36, 39]. Die Wirkung von Oxytocin umschließt folglich die Senkung der CRF-induzierten Effekte zum einen im PVN, zum anderen in der zentralen Amygdala. In beiden Fällen erfolgt die Inhibierung der CRF-Neuronen indirekt über zwischengeschaltete GABAerge Interneurone. Dies führt zur Dämpfung der HPA-Achse und der damit verbundenen Dämpfung der Angstreaktionen. Somit wirkt Oxytocin anxiolytisch und stressreduzierend. Auch die vielfach beschriebenen prosozialen Effekte von Oxytocin können zum Teil mit dessen anxiolytischer Wirkung in Verbindung stehen [17].

Symptome, die durch Oxytocin gelinderten werden können, sind auch diejenigen, die frühere Alkoholabstinenzphase kennzeichnen. Abstinente Alkoholpatienten zeigen Stresssymptome wie Schlafstörungen, Angst, Reizbarkeit, Depression und Konzentrationsstörungen $[1,2]$.

Auch in der Entstehung der Sucht spielen Stress und Angst eine zentrale Rolle. Die Eskalation des Substanzkonsums bei Abhängigkeitserkrankungen wird durch folgende, derzeit gängige 5 Modelle erklärt [40]:

- Toleranzentwicklung gegenüber den Suchtmitteleffekten (Gewöhnung, „Abstumpfung“),

- Sensibilisierung für die Drogeneffekte,

- Allostase, 
Abb. 3 Die hypothetischen Effekte von Oxytocin auf die zentralen Mechanismen der Alkoholabhängigkeit: Oxytocin hemmt über GABA-Interneurone die CRF-Freisetzung, wodurch der Teufelskreis aus immer wiederkehrendem Craving, Alkoholkonsum und Angst bzw. Stress durchbrochen wird. Angriffspunkte sind die zentrale Amygdala sowie die HPA-Achse ausgehend vom PVN. ACTH adrenocorticotropes Hormon, $C R F$ Corticotropin-releasing Factor, GABA $\gamma$-Aminobuttersäure, OXT Oxytocin, PVN Nucleus paraventricularis



Psychologische Forschungsergebnisse konnten zeigen, dass Stresscoping ein validiertes Mittel in der Suchtbehandlung ist [44]. In einer Studie zeigten detoxifizierte Alkoholpatienten Cravingphänomene bei der Präsentation alkoholassoziierter Reize, was im fMRI mit einer Aktivierung der Amygdala einherging. Die Kontrollgruppe dagegen zeigte keinerlei derartige Veränderungen [45]. Nach 3-wöchiger Verhaltenstherapie und Gabe von Antidepressiva zeigten die Testpersonen ein deutlich gesenktes Craving. Die Amygdalaaktivität im fMRI war nun nicht mehr abweichend von der Aktivität der Kontrollpersonen [45]. Diese Befunde unterstreichen die These, dass die Beeinflussung von Stress und die Senkung der CRF-Aktivität in der Amygdala durch Oxytocin zielführend sein könnten.

Neben dem zentralen Kern zeigten sich sowohl in der basolateralen Amygdala als auch der medialen Amygdala im Alkoholentzug Veränderungen der CRFRezeptorexpression [27]. Da dieser Übersichtsartikel auf die zentrale Amygdala fokussiert, wurde auf weiterführende Details der CRF-Regulation der Amygdala, welche sich komplex gestalten und Gegenstand der derzeitigen Forschung sind, nicht weiter eingegangen.

Die bislang einzige Studie, welche den Effekt von Oxytocin im akuten Entzug bei menschlichen Probanden untersuchte, konnte zeigen, dass Oxytocin Craving und Angst senken konnte. Der Clinical-Institute-Withdrawal-Assessment (CIWA)-Score, welcher die klinischen Symptome des Alkoholentzugs quantifiziert, fiel in der mit Oxytocin behandelten Gruppe signifikant niedriger aus. Außerdem konnte durch den Einsatz von Oxytocin die Menge des verabreichten Lorazepam um $80 \%$ reduziert werden. Dazu wurden zweimal pro Tag 24 IU Oxytocin intranasal verabreicht. Limitierend für die Aussagekraft der Studie ist jedoch die geringe Anzahl von lediglich 11 Testper- 
sonen [46]. Dennoch bestätigt dies, dass Oxytocin synergistisch mit Benzodiazepinen wirkt. Benzodiazepine wirken postsynaptisch am GABA-Rezeptor als GABA-A-Agonisten, während Oxytocin präsynaptisch über verstärkte GABA-Ausschüttung wirkt [37]. In unterschiedlichen Tests war der anxiolytische Effekt von Oxytocin mit dem eines Benzodiazepins vergleichbar [47]. Oxytocin bewirkt ebenso wie ein Benzodiazepin Anxiolyse, nach 10 Tagen trat jedoch bei Diazepam in einer Tierstudie ein Gewöhnungseffekt ein, der bei Oxytocin nicht zu verzeichnen war [48]. Wenn dieser ausbleibende oder im Vergleich $\mathrm{zu}$ Benzodiazepinen schwächere Gewöhnungseffekt in weiteren Studien bestätigt wird, bleibt festzustellen, ob diese Eigenschaft auch eine potenzielle Abhängigkeitsentwicklung von Oxytocin beeinflussen würde.

Baclofen, ein GABA-B-Agonist, kann sowohl im akuten Alkoholentzug eingesetzt werden als auch Cravingsymptome bei abstinenten alkoholabhängigen Patienten abschwächen. Es wird spekuliert, dass sich die Wirkung von Baclofen durch die Aktivierung von Oxytocinneuronen im PVN entfaltet $[49,50]$.

In einer weiteren Tierstudie wurden Ratten mit Oxytocin vorbehandelt und in 30 Tagen an Alkohol gewöhnt. In der Gewöhnungsphase zeigte sich kein Unterschied zur Kontrollgruppe im Alkoholkonsum. Nach 20 Tagen, in denen die Ratten freien Zugang zu Alkohol hatten, konsumierten die mit Oxytocin vorbehandelten Ratten signifikant weniger Alkohol als die Kontrollgruppe. Außerdem konnte durch eine weitere Gabe von Oxytocin die konsumierte Alkoholmenge fast halbiert werden. Des Weiteren legte die Oxytocingruppe reduziertes Angstverhalten sowie prosoziales Verhalten an den Tag [51].

$\mathrm{Zu}$ vergleichbaren Ergebnissen kamen Peters et al. [52], welche ebenfalls zeigten, dass i.c.v.-Gabe von Oxytocin bei Ratten im Vergleich zur Kontrollgruppe die konsumierte Alkoholmenge verringerte sowie die Alkoholpräferenz gegenüber Wasser senkte.

\section{Oxytocin in klinischen Studien}

Oxytocin wird in der Gynäkologie schon seit langem sicher eingesetzt, um die Uteruskontraktion und die Laktation zu fördern [53]. Oxytocin besitzt aufgrund des Abbaus durch Aminopeptidasen eine kurze Halbwertszeit von $20 \mathrm{~min}$ im Gehirn bzw. $5 \mathrm{~min}$ in der peripheren Blutzirkulation [54]. Außerdem ist es kaum ZNS-gängig. Dies limitiert den Einsatz von Oxytocin als zentral wirksames Therapeutikum bei peripherer Gabe, da Oxytocin die Blut-Hirn-Schranke nicht in nennenswertem Maße überqueren kann [33, 54]. Zudem konnten nach i.v. Gabe von radioaktiv markiertem Vasopressin, ein Nonapeptid mit gleicher Bioverfügbarkeit, das sich in zwei Aminosäuren von Oxytocin unterscheidet, keine intakten Peptide im Liquor nachgewiesen werden [33]. Bei oraler Gabe würden das saure Milieu und die Enzyme des Magens das kurze Peptidhormon binnen kürzester Zeit inaktivieren.
Um dies zu umgehen wurde Oxytocin in Studien intranasal verabreicht [54]. Die Wirksamkeit von intranasal appliziertem Oxytocin ist belegt [17], der Weg über die Blut-Hirn-Schranke bleibt jedoch unklar. Es bestehen mehrere postulierte Aufnahmewege. Zum einen wird vermutet, dass Oxytocin in olfaktorischen und trigeminalen Neuronen aufgenommen wird, um über axonalen Transport und Exozytose das Gehirn zu erreichen. Jedoch ist fraglich, ob ein Peptid die Internalisierung in Neurone überstehen kann. Zudem benötigt ein axonaler Transport bis hin zum Gehirn Stunden, was im Widerspruch zu den in Studien angesetzten, deutlich kürzeren Zeitfenstern von meist 20-75 min steht. Zum anderen besteht die Überlegung, dass über Interzellulärspalten in der Arachnoidea ein Übertritt von Oxytocin in den Liquor stattfinden könnte. Jedoch weist die Arachnoidea zwischen den Zellen undurchlässige Tight Junctions auf. Ein großes Konzentrationsgefälle nach intranasaler Applikation von Oxytocin könnte jedoch den unspezifischen Transport in den Liquor ermöglichen. Eine weitere Möglichkeit besteht darin, dass Oxytocin intranasal verabreicht die endogene Oxytocinausschüttung anregt $[33,54]$. Zudem ist nicht geklärt, ob ein nachweisbarer Anstieg im Liquor ein geeigneter Messparameter für die Wirksamkeit von Oxytocin nach intranasaler Gabe ist. In verschiedenen Studien erreichten lediglich 0,002-0,005\% des intranasal verabreichten Oxytocin das Gehirn. Außerdem besteht keine Klarheit über die erforderliche applizierte Wirkstoffmenge, um einen Effekt zu erzielen [33, 55]. In den meisten Studien wurden zwischen 8 und 40 IU Oxytocin intranasal verabreicht; dabei wurde über keine Nebenwirkungen berichtet [33, 55, 56]. Trotz der belegten, durch Oxytocin hervorgerufenen Effekte bleibt der Mechanismus von intranasaler Gabe bis zur Wirkungsentfaltung ungeklärt und muss weiter untersucht werden [55]. Ob die intranasale Wirkstoffapplikation von Oxytocin überhaupt den geeignetsten Weg darstellt, um einen Effekt im Gehirn zu erzielen, muss weiter analysiert werden. Darüber hinaus zeigten Carson et al. [57] auf, dass zukünftige Studien methodologisch robuster konzipiert sein sollten, mit dem Ziel, deren statistische Aussagekraft zu erhöhen und bisherige Forschungsergebnisse reproduzierbar zu machen (oder in ihrer Aussagekraft zu überprüfen). Dies erscheint in Anbetracht der eingeschränkten Übertragbarkeit von Grundlagenforschung auf die Anwendung beim Menschen in besonderem Maße von Wichtigkeit zu sein.

Alternativ zum Peptidhormon Oxytocin sind synthetische Small-Molecule-Agonisten, welche keine Peptide sind, partielle Agonisten oder Rezeptormodulatoren denkbar, welche die Einschränkungen des Oxytocins umgehen [58]. Die Mehrzahl der Studien beschäftigte sich mit Fragen des Autismus, der Schizophrenie und Angststörungen. Unklar bleibt, wie sich die längerfristige Gabe von Oxytocin auswirkt [53]. Weitere Untersuchungen sind auch nötig, um die Komplexität der Oxytocin-Signalling-Wege besser zu 
verstehen, insbesondere die extrahypothalamischen Effekte abseits der neurohypophysären Freisetzung in den peripheren Blutkreislauf.

\section{Schlussfolgerungen und Perspektiven}

Der hier postulierte Wirkmechanismus von Oxytocin bezieht sich auf die Auswirkungen der Dysregulation des Stresssystems, insbesondere in Abstinenzphasen, was die Vulnerabilität für Stress erhöht. Da Oxytocin als sicher einsetzbares Medikament gilt, werden derzeit dessen Effekte bei verschiedenen Suchterkrankungen intensiv untersucht [59]. Dabei sollten die Beeinflussung der CRF-Systeme durch Oxytocin und dessen Effekte auf angst- und stressassoziierte Suchtmechanismen beleuchtet werden. Zielpunkte von klinischen Folgestudien könnten die Förderung der Abstinenz durch Linderung der Entzugssymptomatik sowie positive Beeinflussung der Auslöser von Rückfällen in die Alkoholabhängigkeit sein, zu denen in erster Linie Stress und Angst zählen. In diesem Zusammenhang wird die Frage des möglichen Suchtpotenzials von Oxytocin bei langfristiger Anwendung eine wichtige Rolle in zukünftigen Studien einnehmen müssen.

Förderung Die vorliegende Studie wurde zum Teil vom Projekt D-151810-011-013 der Medizinischen Universität Innsbruck unterstützt.

Open access funding provided by University of Innsbruck and Medical University of Innsbruck.

Interessenkonflikt T. Faehrmann, G. Zernig und S. Mechtcheriakov geben an, dass kein Interessenkonflikt besteht.

Open Access Dieser Artikel wird unter der Creative Commons Namensnennung 4.0 International Lizenz (http:// creativecommons.org/licenses/by/4.0/deed.de) veröffentlicht, welche die Nutzung, Vervielfältigung, Bearbeitung, Verbreitung und Wiedergabe in jeglichem Medium und Format erlaubt, sofern Sie den/die ursprünglichen Autor(en) und die Quelle ordnungsgemäß nennen, einen Link zur Creative Commons Lizenz beifügen und angeben, ob Änderungen vorgenommen wurden.

\section{Literatur}

1. Breese GR, Chu K, Dayas CV, Funk D, Knapp DJ, Koob GF, et al. Stress enhancement of craving during sobriety: a risk for relapse. Alcohol Clin Exp Res. 2005;29(2):185-95.

2. Menzaghi F, Rassnick S, Heinrichs S, Baldwin H, Pich EM, Weiss F, etal. The role of corticotropin-releasing factor in the anxiogenic effects of ethanol withdrawal. Ann N YAcad Sci. 1994;739(1):176-84.

3. Zernig G, Kurz M, Saria A, O’Malley SS. Handbuch Alkoholismus. Innsbruck: Verlag der Universitätsklinik für Psychiatrie; 2000.

4. VukovićO, CvetićT,ZebićM, MarićN, BritvićD, Damjanović A, et al. Contemporary framework for alcohol craving. Psychiatr Danub. 2008;20(4):500-7.

5. WHO. ICD-10 Version:2016 2016. http://apps.who.int/ classifications/icd10/browse/2016/en. Zugegriffen: 3. Nov 2015.
6. Sarnyai Z, Shaham Y, Heinrichs SC. The role of corticotropin-releasing factor in drug addiction. Pharmacol Rev. 2001;53(2):209-44.

7. Koob GF. Brain stress systems in the amygdala and addiction. Brain Res. 2009;1293:61-75.

8. Valdez GR, Zorrilla EP, Roberts AJ, Koob GF. Antagonism of corticotropin-releasing factor attenuates the enhanced responsiveness to stress observed during protracted ethanol abstinence. Alcohol. 2003;29(2):55-60.

9. Valdez GR, Roberts AJ, Chan K, Davis H, Brennan M, Zorrilla EP, et al. Increased ethanol self-administration and anxietylike behavior during acute ethanol withdrawal and protracted abstinence: regulation by corticotropin-releasing factor. Alcohol Clin Exp Res. 2002;26(10):1494-501.

10. Breese GR, Sinha R, Heilig M. Chronic alcohol neuroadaptation and stress contribute to susceptibility for alcohol craving and relapse. Pharmacol Ther.2011;129(2):149-71.

11. Garland EL, Boettiger CA, Howard MO. Targeting cognitive-affective risk mechanisms in stress-precipitated alcohol dependence: an integrated, biopsychosocial model of automaticity, allostasis, and addiction. Med Hypotheses. 2011;76(5):745-54.

12. Fisher S, Reason J. (Hrsg.) Handbook of life stress, cognition and health. Oxford: John Wiley \& Sons; 1988.

13. Koob GF, Le Moal M. Drug addiction, dysregulation of reward, and allostasis. Neuropsychopharmacology. 2001;24(2):97-129.

14. Sinha R, Fox HC, Hong KA, Bergquist K, Bhagwagar Z, Siedlarz KM. Enhanced negative emotion and alcohol craving, and altered physiological responses following stress and cue exposure in alcohol dependent individuals. Neuropsychopharmacology. 2009;34(5):1198-208.

15. Onaka T, Takayanagi Y, Yoshida M. Roles of oxytocin neurones in the control of stress, energy metabolism, and social behaviour. J Neuroendocrinol. 2012;24(4):587-98.

16. Gray TS. Amygdaloid CRF pathways: role in autonomic, neuroendocrine, and behavioral responses to stress. Ann N YAcad Sci. 1993;697(1):53-60.

17. Meyer-Lindenberg A, Domes G, Kirsch P, Heinrichs M. Oxytocin and vasopressin in the human brain: social neuropeptides for translational medicine. Nat Rev Neurosci. 2011;12(9):524-38.

18. Muigg P, Scheiber S, Salchner P, Bunck M, Landgraf R, Singewald N. Differential stress-induced neuronal activation patterns in mouse lines selectively bred for high, normal or low anxiety. PLOS ONE. 2009;4(4):e5346.

19. Asede D, Bosch D, Lüthi A, Ferraguti F, Ehrlich I. Sensory inputs to intercalated cells provide fear-learning modulated inhibition to the basolateral amygdala. Neuron. 2015;86(2):541-54.

20. Busti D, Geracitano R, Whittle N, Dalezios Y, Mańko M, Kaufmann W, et al. Different fear states engage distinct networks within the intercalated cell clusters of the amygdala. J Neurosci.2011;31(13):5131-44.

21. Bienvenu TCM, Busti D, Micklem BR, Mansouri M, Magill PJ, Ferraguti F, et al. Large intercalated neurons of amygdala relay noxious sensory information. J Neurosci. 2015;35(5):2044-57.

22. Huber D, Veinante P, Stoop R. Vasopressin and oxytocin excite distinct neuronal populations in the central amygdala. Science. 2005;308(5719):245-8.

23. Barton RA, Aggleton JP, Grenyer R. Evolutionary coherence of the mammalian amygdala. Proc R Soc Lond, B, Biol Sci. 2003;270(1514):539-43.

24. Paxinos G, Watson C. The rat brain in stereotaxic coordinates, 2007, 6. Aufl. London:Academic Press 
25. Alheid GF, Heimer L. New perspectives in basal forebrain organization of special relevancefor neuropsychiatric disorders: the striatopallidal, amygdaloid, and corticopetal components of substantia innominata. Neuroscience. 1988;27(1):1-39.

26. KoobGF.Aroleforbrain stresssystemsinaddiction. Neuron. 2008;59(1):11-34.

27. Heilig M, Koob GF. A key role for corticotropin-releasing factor in alcohol dependence. Trends Neurosci. 2007;30(8):399-406.

28. Lee H-J, Macbeth AH, Pagani J, Young WS. Oxytocin: the great facilitator of life. Prog Neurobiol.2009;88(2):127-51.

29. Gimpl G, Fahrenholz F. The oxytocin receptor system: structure, function, and regulation. Physiol Rev. 2001;81(2):629-83.

30. Windle RJ, Shanks N, Lightman SL, Ingram CD. Central oxytocin administration reduces stress-induced corticosterone release and anxiety behavior in rats. Endocrinology. 1997;138(7):2829-34.

31. Heinrichs M, von Dawans B, Domes G. Oxytocin, vasopressin, and human social behavior. Front Neuroendocrinol. 2009;30(4):548-57.

32. Zheng J, Babygirija R, Bülbül M, Cerjak D, Ludwig K, Takahashi T. Hypothalamic oxytocin mediates adaptation mechanism against chronic stress in rats. Am J Physiol Gastrointest Liver Physiol. 2010;299(4):G946-G53.

33. Leng G, Ludwig M. Intranasal oxytocin: myths and delusions (Biol Psychiatry) 2015. http:/ / www.sciencedirect.com/ science/article/pii/S000632231500400X. Zugegriffen: 20. Okt2015.

34. Bülbül M, Babygirija R, CerjakD, Yoshimoto S, Ludwig K, Takahashi T. Hypothalamic oxytocin attenuates CRF expression via GABAA receptors in rats. Brain Res. 2011;1387:39-45.

35. Smith AS, Tabbaa M, Lei K, Eastham P, Butler MJ, Linton L, et al. Local oxytocin tempers anxiety by activating GABAA receptors in the hypothalamic paraventricular nucleus. Psychoneuroendocrinology. 2016;63:50-8.

36. van den BurgE, Neumann I. Bridging the gap between GPCR activation and behaviour: oxytocin and prolactin signalling in the hypothalamus. J Mol Neurosci. 2011;43(2):200-8.

37. Viviani D, TerrettazT, Magara F, Stoop R. Oxytocin enhances the inhibitory effects of diazepam in the rat central medial amygdala. Neuropharmacology. 2010;58(1):62-8.

38. Knobloch HS, Charlet A, Hoffmann LC, Eliava M, Khrulev S, Cetin AH, et al. Evoked axonal oxytocin release in the central amygdala attenuates fear response. Neuron. 2012;73(3):553-66.

39. SwansonLW, Sawchenko PE, RivierJ, ValeWW. Organization of ovine corticotropin-releasing factor immunoreactive cells and fibers in the rat brain: an Immunohistochemical study. Neuroendocrinology. 1983;36(3):165-86.

40. Zernig G, Ahmed SH, Cardinal RN, Morgan D, Acquas E, Foltin RW, et al. Explaining the escalation of drug use in substance dependence: models and appropriate animal laboratory tests. Pharmacology. 2007;80(2-3):65-119.

41. Anton RF.Whatis craving? AlcoholResHealth. 1999;23(3):165

42. Koob GF. Animal models of craving for ethanol. Addiction. 2000;95(Suppl2):73-81.
43. Koob GF. Alcoholism: allostasis and beyond. Alcohol Clin Exp Res. 2003;27(2):232-43.

44. Sinha R. The role of stress in addiction relapse. Curr Psychiatry Rep. 2007;9(5):388-95.

45. Schneider F, Habel U, Wagner M, Franke P, Salloum JB, Shah NJ, et al. Subcortical correlates of craving in recently abstinent alcoholic patients. Am J Psychiatry. 2001;158(7):1075-83.

46. Pedersen CA, Smedley KL, Leserman J, Jarskog LF, Rau SW, Kampov-Polevoi A, et al. Intranasal oxytocin blocks alcohol withdrawal in human subjects. Alcohol Clin Exp Res. 2013;37(3):484-9.

47. Ring R, Malberg J, Potestio L, Ping J, Boikess S, Luo B, et al. Anxiolytic-like activity of oxytocin in male mice: behavioral and autonomic evidence, therapeutic implications. Psychopharmacology (Berl). 2006;185(2):218-25.

48. Mak P, Broussard C, Vacy K, Broadbear JH. Modulation of anxiety behavior in the elevated plus maze using peptidic oxytocin and vasopressin receptor ligands in the rat. JPsychopharmacol (Oxf). 2012;26(4):532-42.

49. Peters S, Slattery DA, Flor PJ, Neumann ID, Reber SO. Differential effects of baclofen and oxytocin on the increased ethanol consumption following chronic psychosocial stress in mice: stress, ethanol consumption and oxytocin. Addict Biol.2013;18(1):66-77.

50. Kovács LG, Sarnyai Z, Szabó G. Oxytocin and addiction: a review. Psychoneuroendocrinology. 1998;23(8):945-62.

51. Bowen MT, CarsonDS, Spiro A, ArnoldJC, McGregor IS. Adolescent oxytocin exposure causes persistent reductions in anxiety and alcohol consumption and enhances sociability in rats. PLOS ONE. 2011;6(11):e27237.

52. Peters ST, Bowen MT, Bohrer K, McGregor IS, Neumann ID. Oxytocin inhibits ethanol consumption and ethanol-induced dopamine release in the nucleus accumbens. Addict Biol.2016;22(3):702-11.doi:10.1111/adb.12362.

53. MacDonald K, Feifel D. Helping oxytocin deliver: considerations in the development of oxytocin-based therapeutics for brain disorders (Front Neurosci) 2013. http://www. ncbi.nlm.nih.gov/pmc/articles/PMC3597931/ (Erstellt: 15. März2013).Zugegriffen:20. Okt2013.

54. McGonigle P. Peptide therapeutics for CNS indications. Biochem Pharmacol.2012;83(5):559-66.

55. Quintana DS, Woolley JD. Intranasal oxytocin mechanisms can be better understood, but its effects on social cognition and behavior are not to be sniffed at (Biol psychiatry) 2015. http://www.sciencedirect.com/science/article/ pii/S0006322315005284.Zugegriffen:20. Okt2015.

56. NethertonE, SchatteD. Potentialfor oxytocinusein children and adolescents with mental illness. Hum PsychopharmacolClin Exp. 2011;26(4-5):271-81.

57. Carson DS, Yuan H, Labuschagne I. Improving research standards to restore trust in intranasal oxytocin (Biol Psychiatry) 2015. http://www.sciencedirect.com/science/ article/pii/S0006322315007301.Zugegriffen:20. Okt2015.

58 . Modi ME, Young LJ. The oxytocin system in drug discovery for autism: animal models and novel therapeutic strategies. Horm Behav. 2012;61(3):340-50.

59. Lee MR, Weerts EM. Oxytocin for the treatment of drug and alcohol use disorders. Behav Pharmacol. 2016;27(8):640-8. 